\title{
Effect of Oxysterol-Induced Apoptosis of Vascular Smooth Muscle Cells on Experimental Hypercholesterolemia
}

\author{
Sonia Perales, M. José Alejandre, Rogelio Palomino-Morales, Carolina Torres, \\ Jose Iglesias, and Ana Linares
}

Department of Biochemistry and Molecular Biology I, Faculty of Sciences, University of Granada, Campus Universitario de Fuentenueva, Avenida Severo Ochoa, s/n, 18071 Granada, Spain

Correspondence should be addressed to Ana Linares, analinar@ugr.es

Received 12 February 2009; Revised 24 May 2009; Accepted 7 July 2009

Recommended by Seamus J. Martin

\begin{abstract}
Smooth muscle cells (SMCs) undergo changes related to proliferation and apoptosis in the physiological remodeling of vessels and in diseases such as atherosclerosis and restenosis. Recent studies also have demonstrated the vascular cell proliferation and programmed cell death contribute to changes in vascular architecture in normal development and in disease. The present study was designed to investigate the apoptotic pathways induced by 25-hydroxycholesterol in SMCs cultures, using an in vivo/in vitro cell model in which SMCs were isolated and culture from chicken exposed to an atherogenic cholesterol-rich diet (SMC-Ch) and/or an antiatherogenic fish oil-rich diet (SMC-Ch-FO). Cells were exposed in vitro to 25-hydroxycholesterol to study levels of apoptosis and apoptotic proteins $\mathrm{Bcl}-2, \mathrm{Bcl}-\mathrm{X}_{\mathrm{L}}$ and $\mathrm{Bax}$ and the expression of bcl-2 and $\mathrm{bcl}-\mathrm{x}_{\mathrm{L}}$, genes. The quantitative real-time reverse transcriptase-polymerase chain reaction and the Immunoblotting western blot analysis showed that 25-hydroxycholesterol produces apoptosis in SMCs, mediated by a high increase in Bax protein and Bax gene expression. These changes were more marked in SMC-Ch than in SMC-Ch-FO, indicating that dietary cholesterol produces changes in SMCs that make them more susceptible to 25-hydroxycholesterol-mediated apoptosis. Our results suggest that the replacement of a cholesterol-rich diet with a fish oil-rich diet produces some reversal of cholesterol-induced changes in the apoptotic pathways induced by 25-hydroxycholesterol in SMCs cultures, making SMCs more resistant to apoptosis.
\end{abstract}

Copyright (c) 2009 Sonia Perales et al. This is an open access article distributed under the Creative Commons Attribution License, which permits unrestricted use, distribution, and reproduction in any medium, provided the original work is properly cited.

\section{Introduction}

The dedifferentiation and proliferation/apoptosis of smooth muscle cells (SMCs) in the arterial intima represent one of the changes found in early atherosclerotic lesions, when the disease is still reversible [1-3]. Cultured SMCs from an atherosclerotic plaque showed a greater susceptibility to apoptosis that did not vary with subculture [4]. This finding suggests that stable and cell-intrinsic changes in the expression of proapoptotic or antiapoptotic genes exert a greater control over apoptosis in SMCs than do cellcell interactions or the microenvironment in the plaque, indicating that this susceptibility develops in initial stages of the disease.

The decision phase of apoptosis entails the expression of specific pro- and antiapoptotic genes. If the sum of signals gives apoptosis as a result, the whole protein machinery for destruction is unleashed. The Bcl-2 protein family also regulates the apoptosis pathways to influence cell survival; this family forms heterodimers between apoptosis-inhibiting proteins such as $\mathrm{Bcl}-2, \mathrm{Bcl}-\mathrm{XL}$, and $\mathrm{Al}$, and inducing proteins such as Bax, Bad, Bid, regulating cell survival [5]. The ratio of anti- to proapoptotic proteins determines susceptibility to apoptosis, especially the Bcl-2/Bax ratio [6]. SMCs express low levels of Bcl-2 in vitro and in vivo [4,7]. Although SMCs in an atherosclerotic process show no major changes in bcl-2 expression, the balance between antiapoptotic and proapoptotic proteins can change in favor of the latter and trigger apoptosis, mainly mediated by Bax $[8,9]$.

Cholesterol and its oxides are involved in the initiation of atherosclerosis [10-12]. The most numerous cholesterol oxides found in human atheromatous plaque are 7-hydroxycholesterol and 7-ketocholesterol, while 7ketocholesterol, and 25-hydroxycholesterol are the most 
abundant in the aortic wall of experimental animals after a cholesterol-rich diet and are the most effective to induce cell death, especially 25-hydroxycholesterol [13]. Dietary cholesterol is a risk factor for the development of these atherosclerotic changes [3]. Thus, chickens have been reported to readily develop hypercholesterolemia with a cholesterol-rich diet, producing atherosclerotic lesions in some cases [14-16]. Preliminary studies demonstrated that a 20 -day diet enriched with $5 \%$ cholesterol is sufficient to cause hypercholesterolemia and gives rise to lipid deposits in the main arteries that are similar to the fatty streaks observed in the first stages of atherosclerosis [17].

Because the presence of apoptosis in atherosclerotic lesions can have a major impact on the progression of the disease, characterization of oxysteroid-induced cell death is important to understand the development of atherosclerosis, since it is not clear how cholesterol and its oxides induce apoptosis in SMCs. Therefore, the objective of this study was to investigate the apoptotic pathways induced by oxysterols, using a cell model in which SMCs were exposed to atherogenic factors (cholesterol-rich diet) or antiatherogenic factors (fish oil-rich diet) [18]. These cells were then exposed in vitro to 25-hydroxycholesterol, studying levels of apoptosis and apoptotic proteins $\mathrm{Bcl}-2, \mathrm{Bcl}-\mathrm{X}_{\mathrm{L}}$, and $\mathrm{Bax}$ and the expression of genes that encode the proteins involved in apoptosis, bcl-2, bcl- $\mathrm{x}_{\mathrm{L}}$.

\section{Methods and Materials}

2.1. Animals. The protocol of this study was approved by the Animal Laboratory Service of the University of Granada (Spain) and chickens received humane treatment according to the regulations for Animal Research of the European Union. Newborn White Leghorn male chicks (Gallus domesticus), supplied by the Animal Laboratory Service of the University of Granada, were kept in a chamber with a light cycle from 09.00 to 21.00 hours and controlled temperature of $29-31^{\circ} \mathrm{C}$ and allowed free access to food and water.

2.2. Diet and Treatment. The diet was started at hatching and kept on until the chicks were killed (20 days after). Water was available at all time. None of the chicks died a natural death during the treatment nor developed any illness. Three groups of chicks twenty days old were used, the control diet group (C-group) was kept on a standard diet (Sanders A00) while treated group (Ch-group) was fed on the same diet supplemented with $5 \% \mathrm{w} / \mathrm{w}$ powdered cholesterol mixed homogeneously (Panreac reagent Barcelona, pure grade). The third group (Ch-FO-group) was fed for 10 days with a diet of $5 \%$ cholesterol and then cholesterol diet was withdrawn and the standard diet was supplemented with $10 \%$ of fish (menhaden) oil for 10 days more. Experimental diet was prepared daily to minimize the oxidation deterioration. Standard diet free of cholesterol contained (w/w) 42\% carbohydrate (mainly starch), $3.5 \%$ fat, and $20.5 \%$ protein. Experimental diets were obtained by supplementation to de standard diet with $5 \%$ of cholesterol (Ch diet) and $10 \%$ fish (menhaden) oil (FO diet). Fatty acid composition
TABLE 1: Fatty acid composition of control and experimental diet.

\begin{tabular}{|c|c|c|}
\hline Fatty acid & Control & $+10 \%$ fish oil \\
\hline 14 & 0.8 & 6.6 \\
\hline 16 & 22.3 & 22.3 \\
\hline 18 & 8.6 & 5.8 \\
\hline Total sat. & 31.7 & 34.8 \\
\hline $16: 1 \mathrm{n}-7$ & 3.3 & 9.2 \\
\hline $18: 1 \mathrm{n}-9$ & 32.4 & 19.5 \\
\hline $20: 1 n-9$ & 0.0 & 0.4 \\
\hline Total MUFA & 35.7 & 29.1 \\
\hline $18: 2 n-6$ & 24.6 & 10.7 \\
\hline $20: 2 n-6$ & 2.5 & 2.4 \\
\hline $20: 3 n-6$ & 1.1 & 0.6 \\
\hline $20: 4 n-6$ & 1.6 & 1.4 \\
\hline Total n-6 & 29.8 & 15.1 \\
\hline $18: 3 n-3$ & 0.8 & 0.6 \\
\hline $20: 5 n-3$ & 0.0 & 12.0 \\
\hline $22: 5 n-3$ & 1.7 & 1.6 \\
\hline $22: 6 n-3$ & 0.0 & 6.8 \\
\hline Total n-3 & 2.5 & 21.0 \\
\hline Total PUFA & 32.3 & 36.1 \\
\hline Total unsat. & 68.0 & 65.2 \\
\hline Sat./unsat. & 0.47 & 0.53 \\
\hline Sat./PUFA & 0.98 & 0.96 \\
\hline $20: 5 / 22: 6$ & 0.00 & 1.76 \\
\hline$n-3 / n-6$ & 0.08 & 1.39 \\
\hline
\end{tabular}

Results (\% of fatty acid) are expressed as means of 3 determinations

of analysed diets is given in Table 1 [19]. No significant differences were observed in fatty acid composition of each diet during the experiments. After each treatment, animals were decapitated and the aortic arch was removed. Animals did not suffer at all at any stage of the experiment because our laboratory usually uses the anesthetic ketamine $(60 \mathrm{mg} / \mathrm{kg}$ of body weight) and sodium pentobarbital (50 mg/kg of body weight) according to the regulations of the Animal Research of the European Union. The work has a positive inform from the Ethics Committee of the Granada University. Animals received therapy and euthanasia methods. The research staff had the preparation and the Certification to do experimental work with animals. All the stages of the experiment are adapted to the established protocol.

2.3. Smooth Muscle Cell Culture. SMCs were isolated from the aortic arch of the chicks as described elsewhere [17] with slight modifications and cultured in Dulbecco's Modification of Eagle's Medium (DMEM) supplemented with Dglucose $(4.5 \mathrm{~g} / \mathrm{L})$, L-glutamate $(0.584 \mathrm{~g} / \mathrm{mL})$, antibiotic cocktail composed of penicillin $(100 \mu \mathrm{g} / \mathrm{mL})$ and amphotericin $(0.25 \mu \mathrm{g} / \mathrm{mL})$ (Sigma-Aldrich, Inc.), as well as $10 \%(\mathrm{v} / \mathrm{v})$ fetal bovine serum (FBS). Medium was buffered with bicarbonate and cultures were kept at $37^{\circ} \mathrm{C}$ in humidified atmosphere of $95 \%$ air and $5 \% \mathrm{CO}_{2}$. Medium was renewed three times a week. Secondary cultures were initiated after either low or 
high passages using 0.05\%/0.02\% Trypsin-EDTA solution. All experiments were conducted using 3 or 5 passages. Cells were determined to be vascular SMCs by their hill-andvalley configuration at confluence and positive fluorescence staining for smooth muscle actin and myosin [17].

2.4. 25-Hydroxycholesterol Treatment and Cytotoxicity Assay. Cytotoxicity was analyzed by using the MTT assay (3-(4,5-dimethylthiazol-2-yl)-2,5-diphenyltetrazolium, bromide. Sigma). SMCs were plated in 96-well plates at a density of 25000 cells/well. After adhering during overnight culture, cells were treated for 24 and 48 hours with 25 -hydroxycholesterol $(5-40 \mu \mathrm{g} / \mathrm{mL})$ dissolved in absolute ethanol. The final concentration of ethanol in the culture medium never exceeded $0.8 \%$ and no effect on culture was observed at or below this concentration.

MTT was dissolved in DMEM at a concentration of $5 \mathrm{mg} / \mathrm{mL}$. An amount of this solution equal to $10 \%$ of the culture medium volume was added to cell cultures. After 2 hours, cultures were removed from the incubator and washed with PBS. The formazan crystals were solubilized by adding $2001 \mu \mathrm{L}$ of solubilization solution $(0.05 \mathrm{~N}$ $\mathrm{HCl}$ in isopropanol). Metabolic activity was quantified by subtracting light absorbance at $630 \mathrm{~nm}$ from absorbance at $570 \mathrm{~nm}$.

2.5. Real-Time PCR Analysis. Total RNA was isolated with Tri-Reagent/Trizol (Invitrogen, Ltd, UK). Single-stranded cDNA was synthesized from $4 \mu \mathrm{g}$ total RNA using an Oligo $(\mathrm{dT})_{12-18}$ as primer and PowerScript reverse transcriptase (Clontech Laboratories, Inc., Calif, USA). Real-time PCR was performed with the Fast Start DNA Master SYBR Green I Kit (Roche) and Light Cycler system (Roche). For the Light Cycler reaction, a master mix of the following reaction components was prepared to the indicated final concentration: $12.61 \mu \mathrm{L} \mathrm{H} \mathrm{H}_{2} \mathrm{O}, 2.41 \mu \mathrm{L} \mathrm{MgCl}_{2}(4 \mathrm{mM}), 11$ $\mu \mathrm{L}$ forward primer $(0.5 \mu \mathrm{M}), 11 \mu \mathrm{L}$ reverse primer $(0.5 \mu \mathrm{M})$, and $2.01 \mu \mathrm{L}$ of the Fast Start DNA Master SYBR Green I mix (Roche). The primer sequences used in this study are given in Table 2 and optimized at an annealing temperature of $55^{\circ} \mathrm{C}$. The cDNA of the genes studied in the different samples (treated in vivo and in vitro) were diluted 1 : 100 and amplified to obtain the Cp value for each sample. Light Cycler products of the different gene expressions were analyzed by agarose gel electrophoresis and a Light Cycler melting curve was constructed to test for a single product at the end of each PCR reaction. A mathematical model developed by Pfaffl [20] was used for the relative quantification of bcl-2, bcl- $x_{\mathrm{L}}, \mathrm{c}-\mathrm{myc}$, and p53 mRNA expression in real-time PCR with respect to the reference $\beta$-actin gene transcript. The efficiency of the assay for each studied gene was $\beta$-actin: 1.86; bcl-2: 1.89; bcl-x: 1.9; c-myc: 1.91; p53: 1.81 .

2.6. Immunoblotting. Cell monolayer $\left(1 \times 10^{7}\right.$ cells $)$ was disrupted by incubation with RIPA buffer $(1 \times$ PBS, $1 \%$ Nonidet P- $40,0.5 \%$ sodium deoxycholate, $0.1 \%$ SDS, $0.1 \mathrm{mg} / \mathrm{mL}$
PMSF, $2 \mu \mathrm{g} / \mathrm{mL}$ aprotinin, $0.18 \mathrm{mg} / \mathrm{mL}$ sodium orthovanadate). Protein concentration was determined by using the micro-BCA procedure (Bio-Rad Laboratories, Inc., Calif, USA). Proteins were denatured in $1 \times$ sample buffer with $5 \% 2$-mercaptoethanol at $95^{\circ}$ for 10 minutes. Bcl-2, Bcl- $\mathrm{X}_{\mathrm{L}}$, and Bax protein expression was detected by immunoblotting. Total cell lysate was separated under reducing conditions by $12 \%$ SDS-polyacrylamide gel electrophoresis (Mini Protean II, Bio-Rad). Gel-resolved proteins were then transferred onto a polyvinylidene difluoride membrane (PVDF) using a Mini Trans-Blot Cell apparatus (Bio-Rad). Membranes were blocked for 1 hour at room temperature in blocking reagent (5\% milk power, $0,05 \%$ Tween-20 in TBS or PBS, pH 7.4) and probed at $4^{\circ} \mathrm{C}$ overnight with the primary antibody: anti-Bcl- $\mathrm{X}_{\mathrm{L}}$ (Cell signaling Technology, Inc., Mass, USA) 1 : 1000, anti-Bax 1 : 100 (Santa Cruz Biotechnology, Inc., Santa Cruz, Calif, USA), and anti-Bcl-2 1 : 500 (BD Biosciences, Calis, USA). Primary antibodies were detected using a horseradish peroxidase- (HRP-) conjugated secondary antibody. The immunocomplex was detected with the ECL-Plus kit (Amersham, Buckinghamshire, UK), and the band density was analyzed using QuantiScan software (Biosoft, Cambridge, UK).

2.7. Statistical Analysis. Results are expressed as mean \pm SEM. Data were analyzed by Student's $t$-test when the variability was the same in each group, because the $t$-test assumes that standard deviations of two datasets are equal. A difference between groups was considered significant when the $P$-value was $\leq .05$.

\section{Results}

3.1. 25-Hydroxycholesterol Treatment and Cytotoxicity Assay in the In Vivo/In Vitro Cell Culture Model. To evaluate the cytotoxicity of 25-hidroxycholesterol on SMC-C, SMC$\mathrm{Ch}$, and SMC-Ch-FO cultures, cells in third passage were incubated for 24 hours in 25-hidroxycholesterol at concentrations of $0,5,10,20,40$, and $80 \mu \mathrm{g} / \mathrm{mL}$, were used. The 25-hidroxycholesterol was previously dissolved in the ethanol and was diluted in cultures in order to obtain the concentrations of previous experiments. The concentration of ethanol in cultures never exceeded $0.8 \%$, wich previously verified that it was not toxic for the cells. As Figure 1 shows, the citotoxicity of 25-hidroxycholesterol during 24 hours is the same in the three types of cultures. Even then, a slightly greater viability was observed in the SMC-ChFO cultures with respect to the control. With a concentration of $20 \mu \mathrm{g} / \mathrm{mL}$, the cellular viability is approximately of $50 \%$.

3.2. Bax, Bcl-2, and Bcl- $X_{L}$ Protein Levels in the In Vivo/In Vitro Cell Culture Model. Expression of proteins of the Bcl-2 family of oncogenes (bax, bcl- $x_{\mathrm{L}}$, and bcl-2) was studied (Figure 2). Because of the homodimerization and heterodimerization capacity of $\mathrm{Bax}, \mathrm{Bcl}-\mathrm{X}_{\mathrm{L}}$, and $\mathrm{Bcl}-2$, the relationship between expression levels of $\mathrm{Bcl}-2 / \mathrm{Bax}$ and $\mathrm{Bcl}-\mathrm{X}_{\mathrm{L}} / \mathrm{Bax}$ determines whether the cells will undergo 
TABle 2: Primer sequences and PCR product lengths for $\beta$-actin, bcl-2, and bcl-xl.

\begin{tabular}{lllr}
\hline Gen & forward primer & Reverse primer & length \\
\hline$\beta$-actin & GCTCCGGCAATGTGCAA & AGGTTCATGAGGTAGT & 515 \\
bcl-2 & TACCTGCTTACACTTAGGAA & ATGACTATGATGCGATGGCA & 307 \\
bcl-xl & GGAGGAAGAGGATGAGAA & ATCTCCTTGTCCACG & 383 \\
\hline
\end{tabular}

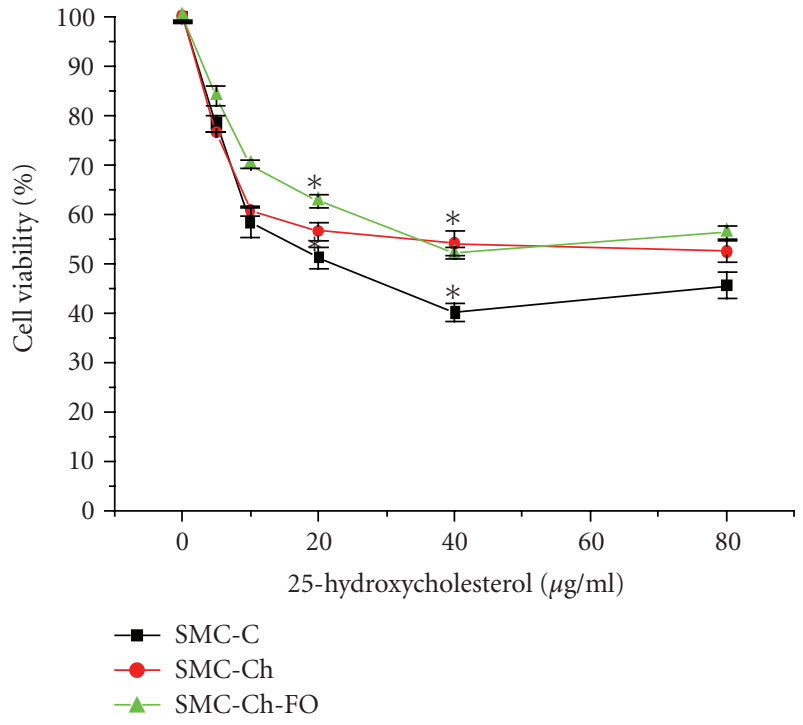

FIGURE 1: Toxicity of 25-hydroxycholesterol to SMC as measured by MTT assay. SMCs were treated with 25-hydroxycholesterol for 24 hours. Lowest values indicate highest toxicity. Results represent mean values $\pm \operatorname{SEM}(n=3) .\left({ }^{*} P \leq .05,{ }^{* *} P \leq .01,{ }^{* * *} P \leq .001\right)$.

apoptosis or not after an apoptotic stimulus. Table 3 shows the antiapoptotic/proapoptotic ratios at baseline (after in vivo treatments) and after 25-hydroxycholesterol in vitro treatment. At baseline, expression of Bcl-2 and Bax (Figure 2) was similar among the three culture types, but expression of Bcl- $\mathrm{X}_{\mathrm{L}}$ was higher in SMC-Ch and SMC-Ch-FO than in $\mathrm{SMC}-\mathrm{C}$. Hence, the Bcl-2/Bax ratios were very similar among cultures, whereas the $\mathrm{Bcl}-\mathrm{X}_{\mathrm{L}} / \mathrm{Bax}$ (Table 3 ) was significantly higher in SMC-Ch-FO. The SMC-Ch-FO (Table 3) showed a highly significant increase in the $\mathrm{Bcl}-\mathrm{X}_{\mathrm{L}} / \mathrm{Bax}$ ratio but a similar Bcl-2/Bax ratio to that of the control cells, indicating some resistance to apoptosis. Therefore, the replacement of a cholesterol-rich diet with a dish oil-rich diet not only reverses the effects of cholesterol but also protects the SMCs from apoptotic stimuli.

3.3. Effect of 25-Hydroxycholesterol on Bax, Bcl-2, and Bcl$X_{L}$ Protein Levels. The addition of 25-hydroxycholesterol produced a highly significant increase in Bax levels in all three culture types (Figure 2) but no change in Bcl- $\mathrm{X}_{\mathrm{L}}$, except for a nonsignificant increase in the SMC-C. Hence, there was a major reduction in the $\mathrm{Bcl}-2 / \mathrm{Bax}$ ratio. Moreover, a large reduction in the $\mathrm{Bcl}-\mathrm{X}_{\mathrm{L}} / \mathrm{Bax}$ ratio was observed for the SMC-C and an intermediate reduction for the SMC-Ch-FO (Table 3).
TABle 3: Bcl-2/Bax and Bcl-xl/Bax expression ratios in SMC cultures: $\mathrm{Bcl}-2 / \mathrm{Bax}$ and $\mathrm{Bcl}-\mathrm{xl} / \mathrm{Bax}$ expression ratios in SMC cultures at baseline (SMC-C, SMC-Ch, and SMC-Ch-FO) and Bcl-2/Bax and Bcl-xl/Bax Expression ratios for SMC cultures treated with $20 \mu \mathrm{g} / \mathrm{ml}$ 25-hydroxycholesterol for 24 hours. Ratios were calculated by using O.D protein and reading and normalized against a control (SMCC) for each protein. Each datum indicated mean \pm SEM of three independent experiments. ${ }^{*} P<.05,{ }^{* *} P<.01$, ${ }^{* * *} P<.001$ versus SMC-C.

\begin{tabular}{|c|c|c|}
\hline & Bcl-X $/$ / Bax & $\mathrm{Bcl}-2 / \mathrm{Bax}$ \\
\hline SMC-C & 1 & 1 \\
\hline SMC-Ch & $1.3^{*}$ & 0.97 \\
\hline SMC-Ch-FO & $1.7^{* *}$ & 1.13 \\
\hline $\begin{array}{l}\text { 25-Hydroxycholesterol } \\
20 \mu \mathrm{g} / \mathrm{ml}\end{array}$ & $\mathrm{Bcl}-\mathrm{X}_{\mathrm{L}} / \mathrm{Bax}$ & Bcl-2/Bax \\
\hline SMC-C & 0.77 & $0.56^{* * *}$ \\
\hline SMC-Ch & 0.99 & $0.61^{* *}$ \\
\hline SMC-Ch-FO & $0.82 * *$ & $0.61^{* *}$ \\
\hline
\end{tabular}

3.4. Expression of bcl-2 and bcl- $X_{L}$ Genes in the In Vivo/In Vitro Cell Culture Model. We also studied the expression of genes that encode proteins implicated in apoptosis (bcl-2, bcl- $\mathrm{x}_{\mathrm{L}}$ ). At baseline (after in vivo experiments), the amount of mRNA of bcl-2 gene remained unchanged in the SMC-C and SMC-Ch (Figure 3). However, the SMC-Ch-FO showed a slight increase in bcl-2 $(P<.05)$. No major differences were observed in baseline expression of the bcl- $\mathrm{x}_{\mathrm{L}}$ gene (Figure 4) in the SMC-C, SMC-Ch, and SMC-Ch-FO cells, as also found for bcl-2 and protein levels.

3.5. Effect of 25-Hydroxycholesterol on the Expression of bcl2 and $b c l-x_{L}$ Genes. Incubation of the cultures with 25hydroxycholesterol increased the mRNA of bcl-2 $(P<.001)$ to a greater degree in SMC-Ch than in SMC-C $(P<.05)$, see Figure 3. Moreover, the expression of bcl- $x_{\mathrm{L}}$ markedly increased in the three culture types after the addition of 25hydroxycholesterol $(P<.001)$, see Figure 4 .

\section{Discussion}

Hyperlipemia and similar conditions, in particular, high cholesterol, change the expression of genes in endothelial cells and SMCs, inducing atherosclerotic lesions in which proliferation and apoptosis are both present $[1,8,21]$. The dedifferentiation and proliferation/apoptosis of SMCs in the arterial intima represent one of the changes found in early atherosclerotic lesions, when the disease is still reversible $[2,3]$. In light of this, it is predictable that SMC culture 


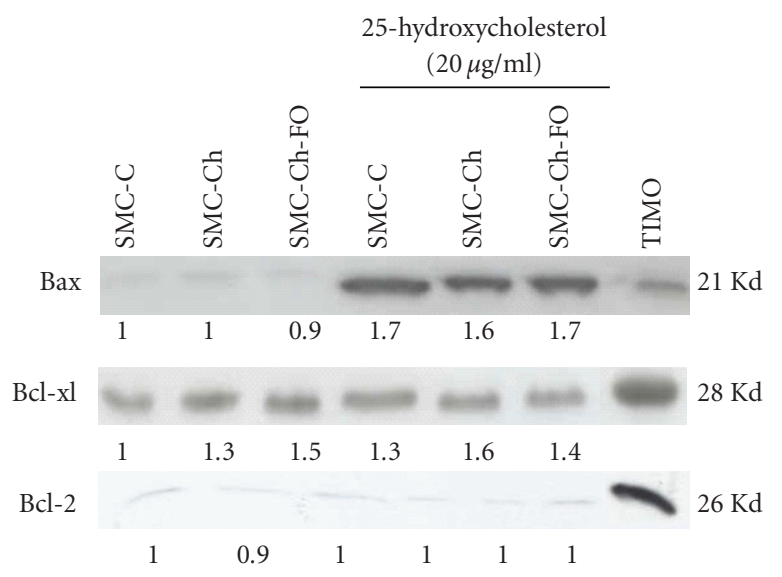

Figure 2: Representative Western blots showing expression levels of Bax, Bcl-xl, and Bcl-2 on SMC cultures at baseline (SMC-C, SMC-Ch, and SMC-Ch-FO) and changes in protein expression after treatment of SMC cultures with $20 \mu \mathrm{g} / \mathrm{mL}$ 25-hydroxycholesterol for 24 hours. Equal amounts of proteins were separated by electrophoresis and processed for immunoblotting. Similar results were obtained in three separate experiments. The ratios were calculated by using O.D protein band reading and normalized against a control (SMC-C) for each protein.

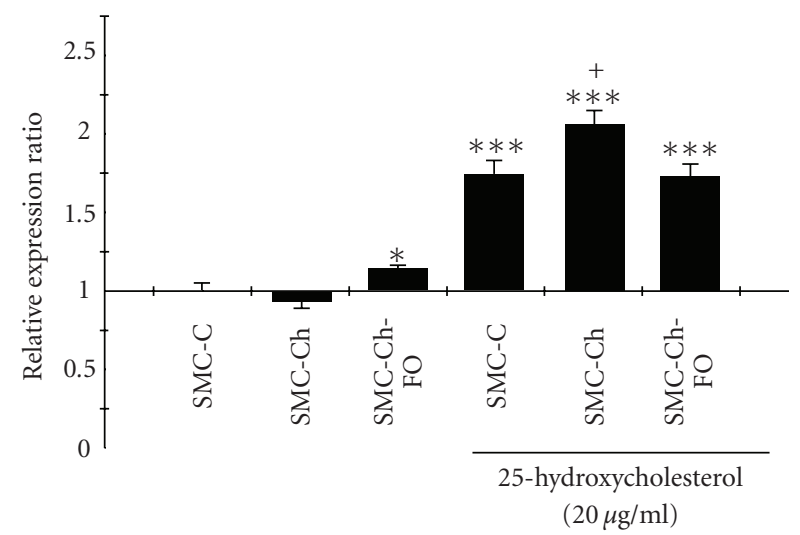

FIGURE 3: bcl-2 mRNA quantification in SMC cultures (SMC-C, SMC-Ch, SMC-Ch-FO) and SMC cultures treated with $20 \mu \mathrm{g} / \mathrm{mL}$ 25-hydroxycholesterol for 24 hours. mRNA levels were quantified by real-time semiquantitative reverse-transcription PCR. Results are shown as relative expression ratio of bcl-2 in SMC cultures with respect to control culture and expressed in comparison to reference gene $\beta$-actin. Bars represent the means \pm SEM for 3 different experiments. ${ }^{*} P<.05,{ }^{* *} P<.01,{ }^{* * *} P<.001$ versus SMC-C $,{ }^{+} P<.05,{ }^{++} P<.01,{ }^{+++} P<.001$ versus SMC-C treated with $20 \mu \mathrm{g} / \mathrm{mL}$ 25-hydroxycholesterol.

models from animals fed with fish oil diet could produce some reversal of the changes induced by cholesterol in apoptotic proteins $\mathrm{Bcl}-2, \mathrm{Bcl}-\mathrm{X}_{\mathrm{L}}$, and $\mathrm{Bax}$ and the expression of bcl-2 and bcl- $x_{\mathrm{L}}$ genes $[22,23]$. The SMC culture model generated in our laboratory by isolating the cells from control and cholesterol-fed chicks [17] produces changes in SMCs that make them more susceptible to 25-hydroxycholesterolmediated apoptosis. Replacement of a cholesterol-rich diet with a fish oil-rich diet produces some reversal of the

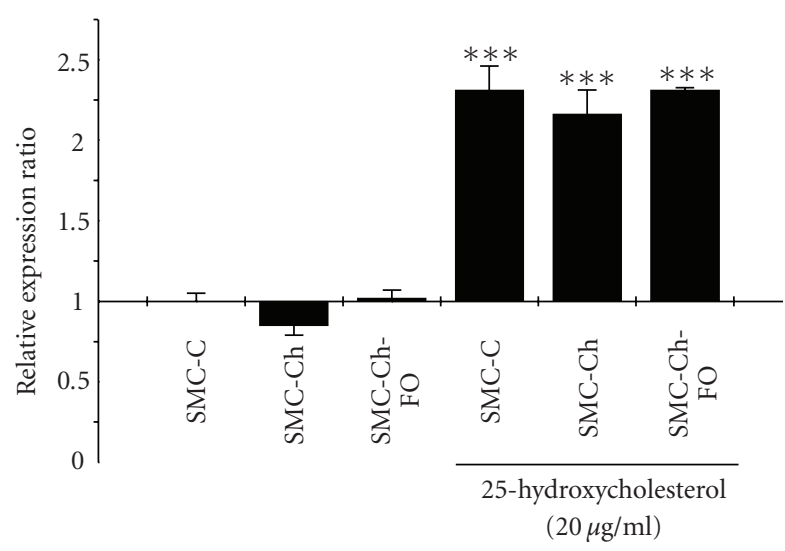

FIGURE 4: bcl-xl mRNA quantification in SMC cultures (SMC-C, SMC-Ch, SMC-Ch-FO) and SMC cultures treated with $20 \mu \mathrm{g} / \mathrm{mL}$ 25-hydroxycholesterol for 24 hours. mRNA levels were quantified by real-time semiquantitative reverse-transcription PCR. Results are shown as relative expression ratio of bcl-2xl in SMC cultures with respect to control culture and expressed in comparison to reference gene $\beta$-actin. Bars represent the means \pm SEM for 3 different experiments. ${ }^{*} P<.05,{ }^{* *} P<.01$, ${ }^{* * *} P<.001$ versus SMC-C $,{ }^{+} P<.05,{ }^{++} P<.01,{ }^{+++} P<.001$ versus SMC-C treated with $20 \mu \mathrm{g} / \mathrm{mL}$ 25-hydroxycholesterol.

cholesterol-induced changes, increasing the resistance of SMCs to apoptosis. The very low level of apoptosis showed in SMCs is similar to the $4 \%$ death rate usually observed in cultured cells. These data are in agreement with previous reports that cultured SMC from the arterial media, even those from an atherosclerotic plaque, showing no apoptosis in culture, unlike observations in SMC from the intima [4]. In early atherosclerosis, the cells in the arterial media that will proliferate and migrate, modulating their phenotype, are in some way protected against apoptosis [24].

In our experimental cell model, Bcl-2 expression was very low in SMCs from chicks after a 20-day calorie-rich diet, whereas Bax expression showed virtually no change. These results are consistent with previous reports that Bcl2 is undetectable in most SMCs from atherosclerotic plaque or healthy arteries $[25,26]$, explaining its poorly defined role, and that the expression of Bax does not differ between SMCs with synthetic and contractile phenotypes. However, other studies suggested that $\mathrm{Bcl}-2$ is expressed in SMCs with contractile phenotype but not in those with synthetic phenotype. It has, therefore, been postulated that Bcl-2 may be repressed throughout the modulation from contractile to synthetic phenotype [27]. This modulation in SMCs of the arterial intima is observed in early atherosclerotic lesions, when the disease is still reversible. Cells in fatty streaks have lipid deposits in small vacuoles in the cytoplasm [27-29]. They subsequently become so-called foam cells [30, 31], and both cell types show a high expression of Bax. Therefore, although the increase in Bax expression in our cells did not reach significance, it suggests that the lesion may still be in an early and reversible state, as are initial adaptive streaks in which $\mathrm{Bax}$ is not detectable [30]. In contrast, the $\mathrm{Bcl}-\mathrm{X}_{\mathrm{L}} / \mathrm{Bax}$ ratio increased in the SMC-Ch. The expression of Bcl- $\mathrm{X}_{\mathrm{L}}$ has 
been reported in SMCs from atherosclerotic plaques, and is believed to play the main role in preventing apoptosis in atherosclerosis [32]. Hence, it is thought to act largely in initial stages of atherosclerosis, in which proliferation must predominate. In fact, an increase in $\mathrm{Bcl}_{-} \mathrm{X}_{\mathrm{L}}$ was observed in SMC from the intima in early atherosclerotic lesions [33]. Other members of the Bcl-2 family, for example, Bak, are abundantly expressed in SMCs from atherosclerotic plaques and tend to heterodimerize with $\mathrm{Bcl}-\mathrm{X}_{\mathrm{L}}$ [33].

Oxysterols, especially 25-hydroxycholesterol, can reduce the expression of bcl-2 y bcl- $x_{L}$ [34-36], activate Bad, and increase the expression of genes that encode $\mathrm{BH} 3$-only proapoptotics, for example, Bim, which in turn activates Bax/Bak. These actions appear to be mediated by the inhibition of AKT survival kinases [37]. Beside the protein levels, we also studied the expression of genes of bcl-2 family that encode proteins implicated in apoptosis (bcl-2, bcl- $\mathrm{x}_{\mathrm{L}}$ ), the amount of mRNA of bcl-2 gene (prototype of antiapoptotic genes) remained unchanged in the SMC-C and SMC-Ch, consistent with previous findings of no modifications in the expression of bcl-2 during atherosclerotic alterations $[9,25$, 26]. However, the SMC-Ch-FO showed a slight increase in bcl-2, which may imply some resistance to apoptotic stimuli. In fact, many genes involved in the response to oxidative stress, for example, NF- $\kappa \mathrm{B}$ survival factor genes, are activated by a fish oil-rich diet and are also involved in the regulation of bcl-2 [38-40]. No major differences observed in baseline expression of the bcl- $\mathrm{x}_{\mathrm{L}}$ gene in the SMC-C, SMC-Ch and SMC-Ch-FO cells, as also found for bcl-2 and protein levels. The bcl- $\mathrm{x}_{\mathrm{L}}$ gene is thought to play a critical role in the prevention of cell death in atherosclerotic plaques, and a reduction in bcl- $\mathrm{x}_{\mathrm{L}}$ expression levels may be a prerequisite for apoptotic induction [9]. It has also been reported that the inhibition of $\mathrm{NF}-\kappa \mathrm{B}$ (activator of cell survival genes reduces the expression of $b c l-\mathrm{x}_{\mathrm{L}}$, which makes the cells more susceptible to apoptosis [41] ).

The increase in the mRNA of bcl-2 to a greater degree in SMC-Ch than in SMC-C may appear contradictory, since Bcl-2 protein levels are known to be reduced by oxysterol treatment of the cells [36]. However, this reduction is largely produced by dimerization of this protein with Bax, which increases in the presence of oxysterols, as demonstrated by the results of our protein studies. The marked increase of the expression of $b c l-x_{L}$ in the three culture types after the addition of 25-hydroxycholesterol again appears contradictory because $\mathrm{Bcl}-\mathrm{X}_{\mathrm{L}}$ protein levels are reduced, which may be explained by the dimerization of $\mathrm{Bcl}-\mathrm{X}_{\mathrm{L}}$ with Bax and especially with Bak [36].

The aortic arches of male chick were chosen for this study for two reasons: first, for the ability of chicks to absorb high amount of dietary cholesterol [14, 42]; and second, because intimal thickening in the aortas from these hypercholesterolemic chicks can be detected after only 20 days [43, 44]. This short diet period is very convenient and contrasts with those reported for other experimental animal models, mainly non-human primates [45] and rabbits [46], which must be a cholesterol-containing diet for between 1 and 18 months to obtain an atherosclerotic lesion. The short time of the chicken model is comparable with that necessary to induce mitotic activity in aortas of white carneau pigeons or swine [47]. Serum cholesterol levels observed in 10-day-old control chicks are normal because newly hatchet chicken reabsorb the yolk during the first week of live [48], thus developing hypercholesterolemia, which becomes almost three times higher in cholesterol-fed chicks. The significantly elevated cholesterol and triglyceride plasma levels are in accordance with previously reported plasma lipids levels [42].

Avian models were used in trials of atherosclerosis, and, in fact, cholesterol feeding has been described elsewhere as producing atherosclerotic lesions in fowl species [16]. We have also used chicks previously to study lipids metabolism after cholesterol administration [49] and to develop a cellculture avian model to look into, in vitro, the effect of a cholesterol diet, in vivo, on the transformation of SMC [17]. Thus, in our laboratory, we isolated SMCs from cholesterolfed chicks (SMC-Ch cultures) which are very proliferative in culture compared with SMC isolated from control-fed chicks (SMC-C cultures), and with identical maintenance in culture of the two lines of SMC [17]. In this way, DNA synthesis in the $S$ phase was 4-fold higher and after 20 days of culture, SMC-Ch increased their cholesterol content to double that of SMC-C, giving SMC-Ch cultures under conditions mimicking such a cholesterol diet and a very early atherosclerosis in vivo/in vitro model at the SMC level [17]. We have studied the cholesterol synthesis and HMG-CoA reductase gene expression in these cultures, showing great differences between SMC-C and SMC-Ch [50, 51]. Also we demonstrated the existence of cyclic fluctuations of HMGCoA reductase activity in nonsynchronized SMC cultures not correlated to the cultured feeding and not with the increase of mRNA, suggesting the posttranscriptional modulation of the HMG-CoA reductase and the relationships between HMGCoA reductase activity and cell division [52]. Finally, we have examined the morphological, molecular, and proliferation change in arterial SMC mimicking such a cholesterol diet. Consequentially, this transformed SMC is a good model to study the alterations of the differentiated state of SMC, caused not only by cholesterol-rich diet but also by fish oil-rich diet as well as the apoptotic pathway induced by oxysteroid in SMCs.

\section{Conclusion}

In conclusion, we investigated the apoptotic pathway induced by oxysteroid in SMCs isolated from male chicks exposed to a control diet (SMC-C) and an atherogenic cholesterol-rich diet (SMC-Ch) or an antiatherogenic fish oil-rich diet (SMC-Ch-FO). We found that 25hydroxycholesterol-induced apoptosis in SMCs, mediated by a high increase in Bax protein and bax gene expression. These changes were more evident in SMC isolated from chicks exposed to an atherogenic than to an antiatherogenic one. Thus, we deducted that the replacement of a cholesterol-rich diet with a fish-oil rich diet might produce some reversal of cholesterol-induced changes in oxysterol-activated apoptotic pathway, making SMC more resistant to apoptosis. Moreover, high cholesterol diet and fish-oil rich diet in 
the animals, before obtaining the SMCs cultures, induced changes in the apoptotic gene expression studied. Thus, the mRNA concentration was downregulated in SMC-ChFO versus SMC-Ch. Since we reported previously that, the nutritional culture conditions, the intracellular cholesterol concentration, and the ultrastructural morphology were the same during the first days of cultures; the change in gene expression must have been induced by the cholesterol or fish oil diet in the aortic SMC in vivo. In this way, this in vivo/in vitro model is relevant to study the nutritional control and gene regulation of the differentiation and apoptosis in SMC. New studies would be necessary to demonstrate the implication of the cell signalling pathways, as well as to show if new protein synthesis is required for the Bax increase or if some peaks of gene expression correlate with other regulatory events.

\section{Acknowledgments}

We would like to thank Maria Romero for her kindly help in the mattering cell cultures and also to the Centro de Instrumentación Cientifica, Flow Citometry Service and Animal Laboratory Service of the University of Granada (Spain). This work was supported partly by Junta de Andalucía (Group code CTS 168) and also by the research grants from Instituto de Salud Carlos III, ISCIII (Grant no. PI030829).

\section{References}

[1] A. Linares, S. Perales, R. J. Palomino-Morales, M. Castillo, and M. J. Alejandre, "Nutritional control, gene regulation, and transformation of vascular smooth muscle cells in atherosclerosis," Cardiovascular and Hematological Disorders, vol. 6, no. 3, pp. 151-168, 2006.

[2] R. Ross, "The pathogenesis of atherosclerosis: a perspective for the 1990s," Nature, vol. 362, no. 6423, pp. 801-809, 1993.

[3] J. H. Campbell and G. R. Campbell, "The role of smooth muscle cells in atherosclerosis," Current Opinion in Lipidology, vol. 5, no. 5, pp. 323-330, 1994.

[4] M. R. Bennett, G. I. Evan, and S. M. Schwartz, "Apoptosis of human vascular smooth muscle cells derived from normal vessels and coronary atherosclerotic plaques," Journal of Clinical Investigation, vol. 95, no. 5, pp. 2266-2274, 1995.

[5] Z. N. Oltvai, C. L. Milliman, and S. J. Korsmeyer, "Bcl-2 heterodimerizes in vivo with a conserved homolog, bax, that accelerates programed cell death," Cell, vol. 74, no. 4, pp. 609619, 1993.

[6] S. J. Korsmeyer, J. R. Shutter, D. J. Veis, D. E. Merry, and Z.N. Oltvai, "Bcl-2/bax: a rheostat that regulates an anti-oxidant pathway and cell death," Seminars in Cancer Biology, vol. 4, no. 6, pp. 327-332, 1993.

[7] M. R. Bennett, G. I. Evan, and S. M. Schwartz, "Apoptosis of rat vascular smooth muscle cells is regulated by p53dependent and -independent pathways," Circulation Research, vol. 77, no. 2, pp. 266-273, 1995.

[8] A. Muto, T. N. Fitzgerald, J. M. Pimiento, et al., "Smooth muscle cell signal transduction: implications of vascular biology for vascular surgeons," Journal of Vascular Surgery, vol. 45, 6, pp. 15-24, 2007.
[9] A. Saxena, J. D. McMeekin, and D. J. Thomson, "Expression of $\mathrm{Bcl}-\mathrm{x}, \mathrm{Bcl}-2$, bax, and Bak in endarterectomy and atherectomy specimens," Journal of Pathology, vol. 196, no. 3, pp. 335-342, 2002.

[10] J. Berliner, N. Leitinger, A. Watson, J. Huber, A. Fogelman, and M. Navab, "Oxidized lipids in atherogenesis: formation, destruction and action," Thrombosis and Haemostasis, vol. 78, no. 1, pp. 195-199, 1997.

[11] G. M. Chisolm and D. Steinberg, "The oxidative modification hypothesis of atherogenesis: an overview," Free Radical Biology and Medicine, vol. 28, no. 12, pp. 1815-1826, 2000.

[12] D. Steinberg, "Low density lipoprotein oxidation and its pathological significance," The Journal of Biological Chemistry, vol. 272, pp. 20963-20966, 1997.

[13] J. Yin, X. Chaufour, C. McLachlan, et al., "Apoptosis of vascular smooth muscle cells induced by cholesterol and its oxides in vitro and in vivo," Atherosclerosis, vol. 148, no. 2, pp. 365-374, 2000.

[14] M. A. E. Mol, R. C. De Smet, A. H. M. Terpstra, and C. E. West, "Effect of dietary protein and cholesterol on cholesterol concentration and lipoprotein pattern in the serum of chickens," Journal of Nutrition, vol. 112, no. 6, pp. 10291037, 1982.

[15] N. S. Moss and E. P. Benditt, "The ultrastructure of spontaneous and experimentally induced arterial lesions. 3. The cholesterol-induced lesions and the effect of a cholesterol and oil diet on the preexisting spontaneous plaque in the chicken aorta," Laboratory Investigation, vol. 23, no. 5, pp. 521-535, 1970.

[16] A. Lucas, W. Yue, X. Y. Jiang, et al., "Development of an avian model for restenosis," Atherosclerosis, vol. 119, no. 1, pp. 1741, 1996.

[17] A. Carazo, M. J. Alejandre, R. Diaz, A. Rios, M. Castillo, and A. Linares, "Changes in cultured arterial smooth muscle cells isolated from chicks upon cholesterol feeding," Lipids, vol. 33, no. 2, pp. 181-190, 1998.

[18] E. Faggin, M. Puato, A. Chiavegato, R. Franch, P. Pauletto, and S. Sartore, "Fish oil supplementation prevents neointima formation in nonhypercholesterolemic balloon-injured rabbit carotid artery by reducing medial and adventitial cell activation," Arteriosclerosis, Thrombosis, and Vascular Biology, vol. 20, no. 1, pp. 152-163, 2000.

[19] M. Castillo, F. Amalik, A. Linares, and E. García-Peregrín, "Dietary fish oil reduces cholesterol and arachidonic acid levels in chick plasma and very low density lipoprotein," Molecular and Cellular Biochemistry, vol. 200, no. 1-2, pp. 5967, 1999.

[20] M. W. Pfaffl, "A new mathematical model for relative quantification in real-time RT-PCR," Nucleic Acids Research, vol. 29, no. 9, p. e45, 2001.

[21] N. J. McCarthy and M. Bennett, "The regulation of vascular smooth muscle cell apoptosis," Cardiovascular Research, vol. 45, no. 3, pp. 747-755, 2000.

[22] M. Ohtsuka, Y. Miyashita, and K. Shirai, "Lipids deposited in human atheromatous lesions induce apoptosis of human vascular smooth muscle cells," Journal of Atherosclerosis and Thrombosis, vol. 13, no. 5, pp. 256-262, 2006.

[23] M. Clarke and M. Bennett, "The emerging role of vascular smooth muscle cell apoptosis in atherosclerosis and plaque stability," American Journal of Nephrology, vol. 26, no. 6, pp. 531-535, 2007. 
[24] M. Mayr and Q. Xu, "Smooth muscle cell apoptosis in arteriosclerosis," Experimental Gerontology, vol. 36, no. 7, pp. 969-987, 2001.

[25] W.-J. Cai, B. Devaux, W. Schaper, and J. Schaper, "The role of Fas/APO 1 and apoptosis in the development of human atherosclerotic lesions," Atherosclerosis, vol. 131, no. 2, pp. 177-186, 1997.

[26] M. M. Konstadoulakis, G. D. Kymionis, M. Karagiani, et al., "Evidence of apoptosis in human carotid atheroma," Journal of Vascular Surgery, vol. 27, no. 4, pp. 733-739, 1998.

[27] Y. Hayakawa, G. Takemura, J. Misao, et al., "Apoptosis and overexpression of bax protein and bax mRNA in smooth muscle cells within intimal hyperplasia of human radial arteries: analysis with arteriovenous fistulas used for hemodialysis," Arteriosclerosis, Thrombosis, and Vascular Biology, vol. 19, no. 9, pp. 2066-2077, 1999.

[28] M. M. Kockx, G. R. Y. De Meyer, N. Buyssens, M. W. M. Knaapen, H. Bult, and A. G. Herman, "Cell composition, replication, and apoptosis in atherosclerotic plaques after 6 months of cholesterol withdrawal," Circulation Research, vol. 83, no. 4, pp. 378-387, 1998.

[29] C. Dong, J. E. Wilson, G. L. Winters, and B. M. McManus, "Human transplant coronary artery disease: pathological evidence for fas-mediated apoptotic cytotoxicity in allograft arteriopathy," Laboratory Investigation, vol. 74, no. 5, pp. 921931, 1996.

[30] M. M. Kockx and A. G. Herman, "Apoptosis in atherosclerosis: beneficial or detrimental?" Cardiovascular Research, vol. 45, no. 3, pp. 736-746, 2000.

[31] P. M. Yao and I. Tabas, "Free cholesterol loading of macrophages is associated with widespread mitochondrial dysfunction and activation of the mitochondrial apoptosis pathway," Journal of Biological Chemistry, vol. 276, no. 45, pp. 42468-42476, 2001.

[32] S. M. Schwartz, D. deBlois, and E. R. M. O'Brien, "The intima: soil for atherosclerosis and restenosis," Circulation Research, vol. 77, no. 3, pp. 445-465, 1995.

[33] M. J. Pollman, J. L. Hall, M. J. Mann, L. Zhang, and G. H. Gibbons, "Inhibition of neointimal cell bcl-x expression induces apoptosis and regression of vascular disease," Nature Medicine, vol. 4, no. 2, pp. 222-227, 1998.

[34] P. F. Dijkers, K. U. Birkenkamp, E. W.-F. Lam, et al., "FKHRL1 can act as a critical effector of cell death induced by cytokine withdrawal: protein kinase B-enhanced cell survival through maintenance of mitochondrial integrity," Journal of Cell Biology, vol. 156, no. 3, pp. 531-542, 2002.

[35] K. Kirito, T. Watanabe, K.-I. Sawada, H. Endo, K. Ozawa, and N. Komatsu, "Thrombopoietin regulates $\mathrm{Bcl}-x_{L}$ gene expression through Stat5 and phosphatidylinositol 3-kinase activation pathways," The Journal of Biological Chemistry, vol. 277, no. 10, pp. 8329-8337, 2002.

[36] E. Nishio and Y. Watanabe, "Oxysterols induced apoptosis in cultured smooth muscle cells through CPP32 protease activation and bcl-2 protein downregulation," Biochemical and Biophysical Research Communications, vol. 226, no. 3, pp. 928934, 1996.

[37] A. E. Rusiñol, D. Thewke, J. Liu, N. Freeman, S. R. Panini, and M. S. Sinensky, "AKT/protein kinase B regulation of BCL family members during oxysterol-induced apoptosis," Journal of Biological Chemistry, vol. 279, no. 2, pp. 1392-1399, 2004.

[38] M. Magnani, R. Crinelli, M. Bianchi, and A. Antonelli, "The ubiquitin-dependent proteolytic system and other potential targets for the modulation of nuclear factor-kB (NF-kB)," Current Drug Targets, vol. 1, no. 4, pp. 387-399, 2000.
[39] P. C. Rath and B. B. Aggarwal, "TNF-induced signaling in apoptosis," Journal of Clinical Immunology, vol. 19, no. 6, pp. 350-364, 1999.

[40] M. Takahashi, N. Tsuboyama-Kasaoka, T. Nakatani, et al., "Fish oil feeding alters liver gene expressions to defend against PPAR $\alpha$ activation and ROS production," American Journal of Physiology, vol. 282, no. 2, pp. G338-G348, 2002.

[41] M. R. Bennett, G. I. Evan, and A. C. Newby, "Deregulated expression of the c-myc oncogene abolishes inhibition of proliferation of rat vascular smooth muscle cells by serum reduction, interferon-gamma, heparin, and cyclic nucleotide analogues and induces apoptosis," Circulation Research, vol. 74, no. 3, pp. 525-536, 1994.

[42] J. L. Segovia, M. García-Gonzalez, and M. J. Alejandre, "The role of age on the cholesterol-metabolizing enzymes and lipid levels in chick plasma and liver microsomes after cholesterol enriched diet cessation," Biochemistry and Molecular Biology International, vol. 34, no. 1, pp. 93-100, 1994.

[43] R. Díaz, F. J. González, M. Reyes, et al., "Fith creteil symposium on lipids, lipoproteins and nutritiumml: isolation and cultura of vascular smooth muscle cells during an experimental atherosclerosis," Annals of Nutrition and Metabolism, vol. 35, pp. 370-371, 1991.

[44] M. Reyes, F. J. González, R. Díaz, et al., "Fith creteil symposium on lipids, lipoproteins and nutritiumml: proliferation studiesof cultured smooth muscle cells during early stages of an experimental atherosclerosis," Annals of Nutrition and Metabolism, vol. 35, pp. 370-371, 1991.

[45] A. Faggiotto, R. Ross, and L. Harker, "Studies of hypercholesterolemia in the nonhuman primate. I. Changes that lead to fatty streak formation," Arteriosclerosis, vol. 4, no. 4, pp. 323340, 1984.

[46] M. E. Rosenfeld and R. Ross, "Macrophage and smooth muscle cell proliferation in atherosclerotic lesions of WHHL and comparably hypercholesterolemic fat-fed rabbits," Arteriosclerosis, vol. 10, no. 5, pp. 680-687, 1990.

[47] W. G. Jerome and J. C. Lewis, "Early atherogenesis in White Carneau pigeons. I. Leukocyte margination and endothelial alterations at the celiac bifurcation," American Journal of Pathology, vol. 116, no. 1, pp. 56-68, 1984.

[48] R. C. Noble and M. Cocchi, "Lipid metabolism and the neonatal chicken," Progress in Lipid Research, vol. 29, no. 2, pp. 107-140, 1990.

[49] M. J. Alejandre, H. Ramírez, J. L. Segovia, and E. GarcíaPeregrin, "Effect of dietary cholesterol and cholestyramine on developmental pattern of 3-hydroxy-3-methylglutaryl-CoA reductase," Annals of Nutrition and Metabolism, vol. 29, no. 2, pp. 111-118, 1985.

[50] A. Carazo, M. J. Alejandre, A. Louktibi, and A. Linares, "The reversal of the inhibition on lipids synthesis by L-659,699 in arterial smooth muscle cells cultures," Molecular and Cellular Biochemistry, vol. 221, no. 1-2, pp. 25-31, 2001.

[51] A. Carazo, M. J. Alejandre, M. D. Suárez, and A. Linares, "Alterations in 3-hydroxy-3-methylglutaryl-CoA reductase mRNA concentration in cultured chick aortic smooth muscle cells," Lipids, vol. 35, no. 6, pp. 587-593, 2000.

[52] M. J. Alejandre, S. Perales, A. Carazo, R. PalominoMorales, and A. Linares, "Cyclic fluctuations of 3-hydroxy3-methylglutaryl-CoA reductase in aortic smooth muscle cell cultures," Lipids, vol. 41, no. 12, pp. 1089-1099, 2006. 

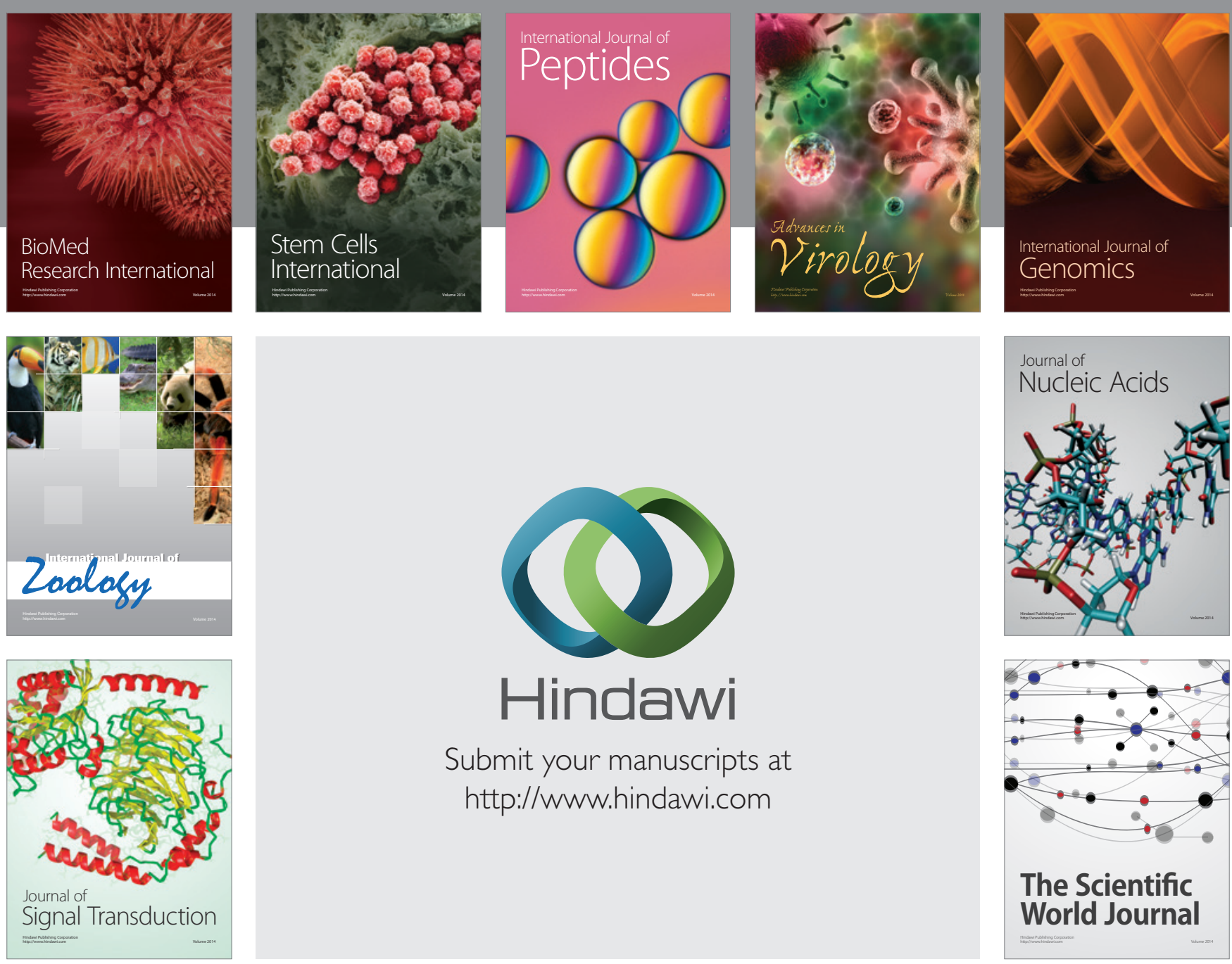

Submit your manuscripts at

http://www.hindawi.com
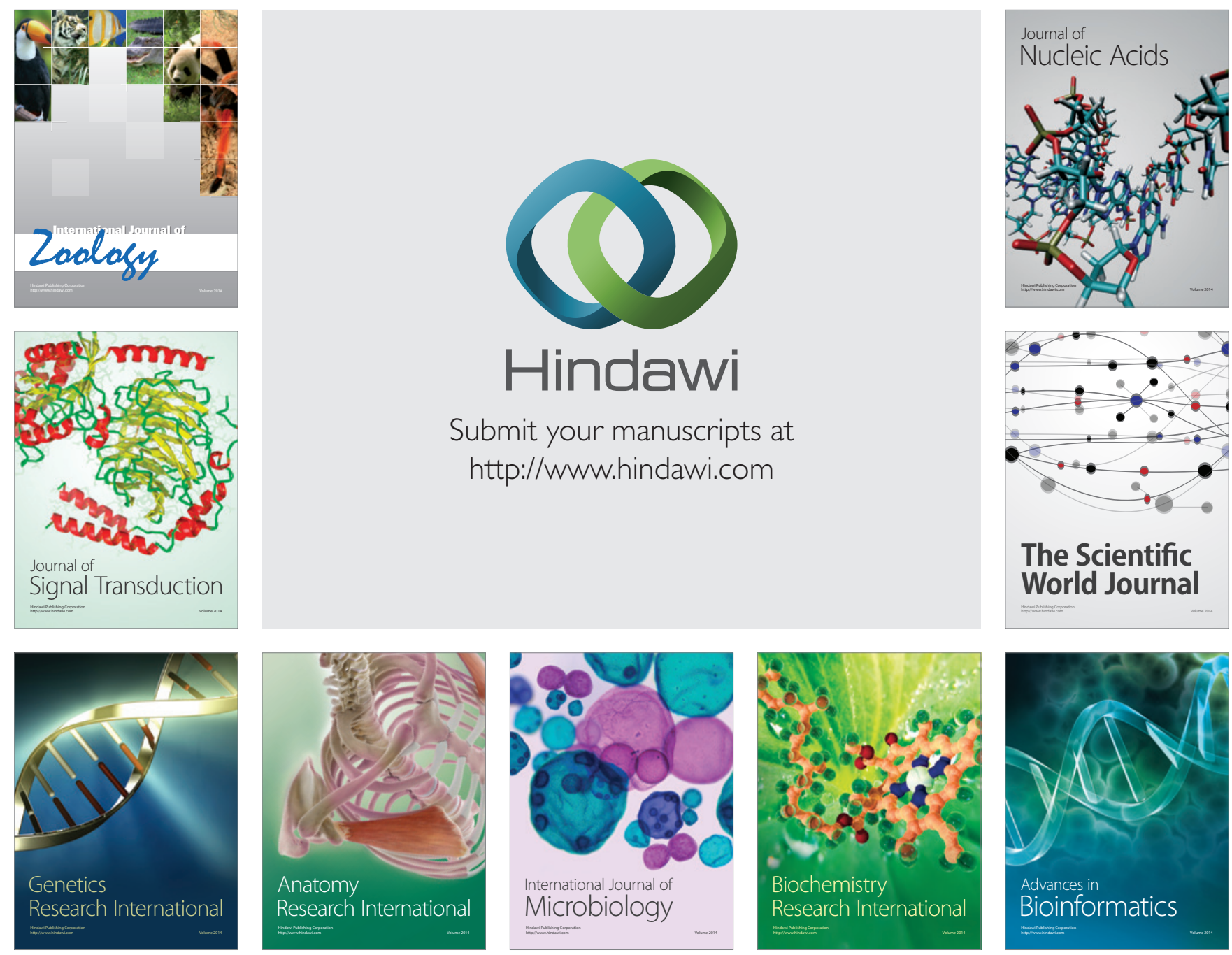

The Scientific World Journal
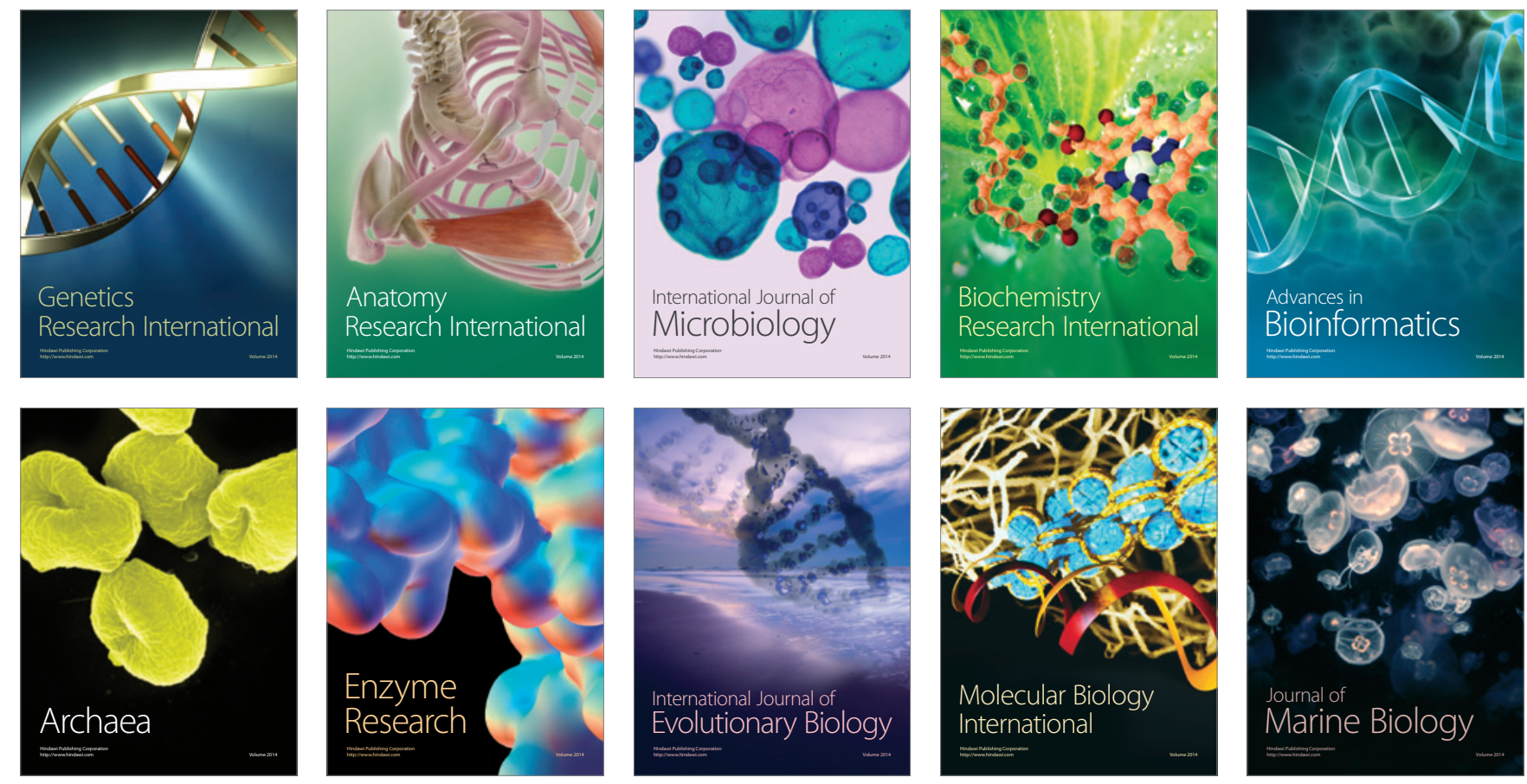